\title{
POINCARE COMPLEX THICKENINGS AND CONCORDANCE OBSTRUCTIONS
}

\author{
BY J. P. E. HODGSON \\ Comunicated by P. Emery Thomas, October 24, 1969
}

0 . Introduction. The object of this note is to announce some results on the problem of concordance classification of piecewise linear and smooth homeomorphisms of manifolds. These results are obtained by using the analogous problem for Poincaré complexes to relate the author's results in [2] to the results obtained by use of the techniques of Sullivan as developed in [6].

Briefly the approach is as follows, we describe the author's and Sullivan's results in terms of relative homotopy groups of certain $\Delta$-sets, these fit naturally into the exact sequence of a triple as "two" of the terms, the "third" term can then be interpreted by use of the concept of a Poincaré complex thickening. We begin with a restatement of known results. In general we shall confine our remarks to the piecewise linear category while indicating the modifications which may be required in the smooth case.

\section{Recall of results.}

Definitions. We recall the definition of the $\Delta$-set [5], $\varepsilon(M)$ of homotopy equivalences of $M$, where $M$ is a PL or smooth manifold with boundary $M$.

A $k$-simplex of $\mathcal{E}(M)$ is a homotopy equivalence $f: \Delta^{k} \times M \rightarrow \Delta^{k} \times M$ such that restriction to a face $\Delta^{s}$ of $\Delta^{k}$ induces a homotopy equivalence of $\Delta^{s} \times M$, and if $D_{*}^{m}$ is a fixed disc containing the base point of $M$ then $f \mid D_{*}^{m} \times D=$ Id.

A $k$-simplex of $D(M)$ is a PL homeomorphism $f: \Delta^{k} \times M \rightarrow \Delta^{k} \times M$ such that $f \mid D_{*}^{m} \times D=$ Id and restriction to a face $\Delta^{s}$ of $\Delta^{k}$ induces a PL homeomorphism of $\Delta^{s} \times M$.

In the differentiable case a slightly more complicated definition is required; the reader is referred to [2] for the details.

We require a third $\Delta$-set, $\varepsilon(M, \partial M)$ a $k$-simplex of which is a homotopy equivalence of pairs

$$
f: \Delta^{k} \times M, \quad \Delta^{k} \times \partial M \rightarrow \Delta^{k} \times M, \quad \Delta^{k} \times \partial M
$$

such that $f \mid D_{*}^{m} \times D=$ Id and restriction to a face $\Delta^{s}$ of $\Delta^{k}$ induces a

AMS 1969 subject classifications. Primary 5568, 5701, 5720.

Key words and phrases. Poincaré complex, thickening, $\Delta$-set, relative homotopy group, homeotopy group. 
homotopy equivalence of the pair $\Delta^{s} \times M, \Delta^{s} \times \partial M$.

We now have (see for example [5] for the discussion of the exact sequence of a triple)

Proposition 1. There is an exact sequence

$$
\begin{aligned}
\rightarrow \pi_{1}(\mathcal{E}(M, \partial M), D(M)) \rightarrow \pi_{1}(\mathcal{E}(M), D(M)) & \rightarrow \pi_{1}(\mathcal{E}(M), \mathcal{E}(M, \partial M)) \\
& \rightarrow \pi_{0}(\mathcal{E}(M, \partial M), D(M)) .
\end{aligned}
$$

and we recall

Theorem A [2]. Let $K$ be a finite connected CW-complex of dimension $k$, which is $(2 k-m+2)$-connected and of the homotopy type of a suspension. If $\phi: K \rightarrow M$ is an m-thickening of $K$, then there exists a bijection

$$
\pi_{1}(\mathcal{E}(M), D(M)) \rightarrow J^{m+1}(S K) .
$$

and

Theorem B (Sullivan [6]. Let $(M, \partial M)$ be a connected and simplyconnected PL manifold with nonempty simply-connected boundary $\partial M$, and suppose that $\operatorname{dim}(M) \geqq 6$ then there is a bijection

$$
\pi_{1}(\varepsilon(M, \partial M), D(M)) \rightarrow[S M, F / \mathrm{PL}] .
$$

The main portion of this note will be taken up with a description of the relative group $\pi_{1}(\mathcal{E}(M), \varepsilon(M, \partial M))$ under certain favourable conditions.

2. Poincaré complex thickenings. The basic concept for our interpretation of $\pi_{1}(\mathcal{E}(M), \mathcal{E}(M, \partial M))$ is that of a Poincaré complex thickening, which we shall now define.

Definition 2.1. Let $K$ be a finite connected CW-complex of dimension $k$, then a Poincaré complex thickening of $K$ of dimension $m$ is a map $f: M^{m-1} \rightarrow K$ where $m \geqq 6, m-k \geqq 3$, and $M^{m-1}$ is a Poincaré complex of dimension $(m-1)$ with fundamental class $[M]$, such that

1. $f_{*}: \pi_{1}(M) \rightarrow \pi_{1}(K)$ is an isomorphism.

2. If $M_{f}$ denotes the mapping cylinder of $f,\left(M_{f}, M\right) \sim(K, M)$ is a Poincaré pair of dimension $m$, with fundamental class $[K, M]$ and such that in the homology exact sequence of the pair $(K, M)$, $\delta[K, M]=[M]$.

We remark that by $[8]$, the class $[M]$ may be regarded as orienting the top dimensional cell of $M$.

Two such thickenings $f_{1}: M_{1} \rightarrow K$ and $f_{2}: M_{2} \rightarrow K$ are equivalent if there exists a homotopy equivalence $k:\left(K, M_{1}\right) \rightarrow\left(K, M_{2}\right)$ of degree 1 , such that the following diagram commutes up to homotopy. 


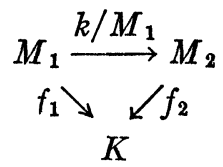

We shall denote the set of such equivalence classes by $J_{h}^{m}(K)$. The Poincaré complex $M$ will be called the boundary of the thickening.

The basic result of this note is the following

Theorem 2.2. Let $f: K^{k} \rightarrow M^{m}$ be a PL-thickening of the finite connected $\mathrm{CW}$-complex $K$, and suppose that $K$ satisfies the following additional hypotheses:

1. $K$ is $(2 k-m+3)$-connected.

2. $K$ is of the homotopy type of a suspension.

Then there is a bijection

$$
\pi_{1}(\mathcal{E}(M), \mathcal{E}(M, \partial M)) \rightarrow J_{h}^{m+1}(S K) .
$$

The proof of this result will appear elsewhere, and depends on the following result.

Theorem 2.3. Let $\left(S^{m}, T^{m-1}\right)$ be a Poincaré pair and let $f: P^{p} \rightarrow S^{m}$ be a $(2 p-m+2)$-connected map, where $P$ is a $(2 p-m+2)$-connected $p$ dimensional finite $\mathrm{CW}$-complex. Then there exists a Poincare complex thickening $g: Q^{m-1} \rightarrow P$ and a Poincarê pair $(Y, Q \cup T)$ such that $Y \cup_{Q} M_{g} \sim S \bmod T$ and $P \rightarrow M_{g} \cup_{Q} Y \rightarrow S$ is homotopic to $f$. Further the homotopy type of such a pair $(P, Q)$ is unique if $(2 p-m+2)$ is replaced by $(2 p-m+3)$ throughout.

This has the following corollary

Corollary 2.4. If $f: K \rightarrow L$ is a $(2 k-m+3)$-connected map between $\mathrm{CW}$-complexes and $K$ is also $(2 k-m+3)$-connecied then $f$ induces

$$
f^{*}: J_{h}^{m}(K) \rightarrow J_{h}^{m}(L)
$$

where $f^{*}$ depends only on the homotopy class of $f$.

There is also a relative version of Theorem 2.3, along the lines of Theorem 2.3 of [2].

3. Applications. We consider the case of manifolds with sphere boundaries. In this case we have the following known result from [3]. (See also [4] for the smooth case.)

Theorem 3.1. Let $M^{m}$ be a thickening of the $\mathrm{CW}$-complex $K$, suppose $\partial M=S^{m-1}$, and that $M$ is $[m / 3]+1$ connected. Then $\operatorname{Im}\left\{\pi_{1}(\varepsilon(M), D(M)) \rightarrow \pi_{0}(D(M))\right\} \cong \operatorname{Im}\left\{J^{m+1}(S K) \rightarrow J^{m+2}(S K)\right\}$. 
Our aim is to use this result to determine the image of

$$
\pi_{1}(\mathcal{E}(M, \partial M), D(M)) \text { in } \pi_{0}(D(M)),
$$

and hence to get information on the homotopy group of the closed manifold obtained from $M$ by filling in the boundary with a disc.

Definition. Define $J T^{m}(K)$ to be the kernel (inverse image of the trivial thickening) of the forgetting map $J^{m}(K) \rightarrow J_{h}^{m}(K)$.

REMARK. In the case where $K$ is a suspension and it is $(2 k-m+3)$ connected this becomes a kernel in the usual sense, as $J^{m}(K)$ can be given a group structure as in the usual case.

With this definition we have

THEOREM 3.2. Let $M$ be a PL (smooth) manifold with boundary a sphere and suppose that $M$ is $(m / 3)+1$ connected. Then

$$
\operatorname{Im}\left\{\pi_{1}(\mathcal{E}(M, \partial M), D(M)) \rightarrow \pi_{0}(D(M))\right\} \cong J T^{m+2}(S K) .
$$

Proof. Consider the diagram

$$
\begin{aligned}
& \pi_{1}(\mathcal{E}(M, \partial M), D(M)) \quad \stackrel{i_{0}}{\rightarrow} \pi_{1}(\mathcal{E}(M), D(M)) \stackrel{j_{0}}{\rightarrow} \quad \pi_{1}(\mathcal{E}(M), \mathcal{E}(M, \partial M)) \\
& \alpha \downarrow \quad \downarrow \beta \quad \downarrow \gamma \\
& \pi_{1}(\mathcal{E}(M \times I, \partial(M \times I)), D(M \times I)) \stackrel{i_{1}}{\rightarrow} \pi_{1}(\mathcal{E}(M), D(M \times I)) \stackrel{j_{1}}{\rightarrow} \pi_{1}(\mathcal{E}(M), \mathcal{L}(M \times I, \partial(M \times I)))
\end{aligned}
$$

then by Theorem B, $\alpha$ is an isomorphism, and we have from Theorem 3.1 that

$$
\operatorname{Im} \beta \cong \operatorname{Im}\left\{\pi_{1}(\mathcal{E}(M), D(M)) \rightarrow \pi_{0}(D(M))\right\}
$$

but $\pi_{1}(\mathcal{E}(M, \partial M), D(M)) \rightarrow \pi_{0}(D(M))$ factors through $\pi_{1}(\mathcal{E}(M), \mathfrak{D}(M))$ by the exact sequences associated to the triple $(\mathcal{E}(M), \mathcal{E}(M, \partial M)$, $D(M))$. Thus

$$
\begin{aligned}
& \operatorname{Im}\left\{\pi_{1}(\varepsilon(M, \partial M), \mathfrak{D}(M)) \rightarrow \pi_{0}(D(M))\right\} \\
& \cong \operatorname{Im}\left\{\pi_{1}(\varepsilon(M \times I, \partial(M \times I)), D(M \times I)) \rightarrow \pi_{1}(\mathcal{D}(M), D(M \times I))\right\}
\end{aligned}
$$

which is by exactness taken together with Theorem A and Theorem 2.2 just $J T^{m+2}(S K)$.

Using this result, information on the $J$-homomorphism [1] and work of Sullivan [7] one can show the following

TheOREM 3.3. Let $M^{m}$ be a closed metastably connected PL manifold such that $H^{4 i-1}(M, Z)$ contains no 2-torsion for any $i$, then if $\operatorname{dim} M \geqq 6$ there is no 2-torsion in the homeotopy group of $M$.

\section{REFERENCES}

1. J. F. Adams, On the groups $J(X)$. IV, Topology 5 (1966), 21-71. MR 33 \#6628.

2. J. P. E. Hodgson, Obstructions to concordance for thickenings, Invent. Math. 5 (1968), 292-316. MR 37 \#5879. 
3. - Automorphisms of metastably connected PL-manifolds, Preprint, University of Pennsylvania, Phil., $\mathrm{Pa}$., 1968.

4. - - Automorphisms of some smooth metastably connected manifolds, Preprint, University of Warwick, Coventry, England, 1969.

5. C. P. Rourke and B. J. Sanderson, $\Delta-$ Sets. I, Preprint, University of Warwick, Coventry. England, 1969.

6. D. Sullivan, Triangulating homotopy equivalences, Preprint, University of Warwick, Coventry, England, 1966.

7. - Geometric topology, Notes, Princeton, 1967.

8. C. T. C. Wall, Poincarê complexes. I, Ann. of Math. (2) 86 (1967), 213-245. MR $36 \# 880$.

University OF WARWICK, Coventry, England 